\title{
Probabilistic low-rank factorization accelerates tensor network simulations of critical quantum many-body ground states
}

\author{
Lucas Kohn, ${ }^{1}$ Ferdinand Tschirsich, ${ }^{1, *}$ Maximilian Keck, ${ }^{2}$ Martin B. Plenio, ${ }^{3}$ Dario Tamascelli, ${ }^{3,4}$ and Simone Montangero ${ }^{1,5,6}$ \\ ${ }^{1}$ Institute for Complex Quantum Systems and Center for Integrated Quantum Science and Technologies, \\ Universität Ulm, 89069 Ulm, Germany \\ ${ }^{2}$ NEST, Scuola Normale Superiore and Istituto Nanoscienze CNR, 56126 Pisa, Italy \\ ${ }^{3}$ Institute for Theoretical Physics, Universität Ulm, 89069 Ulm, Germany \\ ${ }^{4}$ Dipartimento di Fisica, Università degli Studi di Milano, 20133 Milano, Italy \\ ${ }^{5}$ Theoretische Physik, Universität des Saarlandes, 66123 Saarbrücken, Germany \\ ${ }^{6}$ Dipartimento di Fisica e Astronomia, Università degli Studi di Padova, 35131 Padova, Italy
}

(Received 11 October 2017; published 3 January 2018)

\begin{abstract}
We provide evidence that randomized low-rank factorization is a powerful tool for the determination of the ground-state properties of low-dimensional lattice Hamiltonians through tensor network techniques. In particular, we show that randomized matrix factorization outperforms truncated singular value decomposition based on stateof-the-art deterministic routines in time-evolving block decimation (TEBD) - and density matrix renormalization group (DMRG)-style simulations, even when the system under study gets close to a phase transition: We report linear speedups in the bond or local dimension of up to 24 times in quasi-two-dimensional cylindrical systems.
\end{abstract}

DOI: 10.1103/PhysRevE.97.013301

\section{INTRODUCTION}

Tensor network (TN) methods have long proven their power as indispensable tools in simulating quantum and classical many-body systems [1,2]. As first realized by White with the density matrix renormalization group (DMRG) algorithm $[3,4]$, a variational ansatz on the manifold of matrix product states (MPS) [5], TNs provide an efficient parametrization of low-entangled wave functions in quantum many-body state space [6]. While the MPS naturally captures the relevant lowenergy spectrum in particular of one-dimensional (1D) gapped Hamiltonians obeying area laws of entanglement [7-10], TNs have been generalized to more complex scenarios: In over two decades of evolution, they have been successfully applied to higher dimensions [11-13], critical phenomena [14-17], finite temperature and closed- and open-system dynamics $[18,19]$, and lattice gauge theories [20-22], just to name a few examples. TNs have also been equipped with structure to encode and exploit symmetries in the model under investigation [23-26].

Truncated singular value decompositions (SVDs) are widely used in TN algorithms to compress states into their respective TN state manifold. Examples include the timeevolving block decimation (TEBD) $[27,28]$, the tensor renormalization group [29], the corner transfer matrix renormalization group (CTMRG) [30], and their application to projected entangled pair states (PEPS) [31-34], but also traditional DMRG which is often formulated in terms of truncated eigenvalue decomposition. In TN numerical practice, SVDs have the additional advantage of providing relevant isometries by orthonormality of the singular vectors and reveal valuable

\footnotetext{
*ferdinand.tschirsich@uni-ulm.de
}

information of the encoded network state, e.g., in the form of entanglement measures based on singular values.

The traditional way to compute a truncated SVD is to first perform the full SVD of a matrix and then discard the smallest singular values. This is reliable and accurate, but also a very costly operation that often dominates computational complexity of TN algorithms. Intuitively, it is also not the most economic protocol: A lot of effort is spent in computing all singular values and vectors, many of which are then discarded. By avoiding the full SVD, a truncated SVD can be obtained more efficiently, especially when the number of retained singular values is small. Well-known methods of this class are simultaneous subspace iteration or Krylov subspace methods like Lanczos or implicitly restarted Arnoldi algorithms [35,36]. Their relevance in large-scale data classification and compression in "big data" applications [37,38], signal processing [39], face recognition [40,41], DNA analysis [42], and other fields is a driving force behind the ongoing development of faster algorithms. A use case in the approximative contraction of unstructured TNs has also been reported [43].

Randomized algorithms outperform prior approaches in both speed and reliability [44]. Specifically, the randomized SVD (RSVD) based on a probabilistic low-rank matrixfactorization algorithm [44] is capable of delivering accurate results with failure probabilities that can be made arbitrarily small, independent of peculiar choices like starting vectors that are common in deterministic methods. RSVD thus promises to significantly accelerate TN methods that spend a considerable amount of resources in truncated SVDs.

Recently, significant speedup due to RSVD has been reported in the TEBD simulation of open-system dynamics [45]. In particular, the authors of Ref. [45] showed that the robust RSVD outperforms deterministic SVD algorithms in 
delivering a limited number of largest singular values (and corresponding vectors) while maintaining high accuracy in the simulated dynamics. It is, however, an open question of whether RSVD can be applied with similar success in scenarios beyond the open-system dynamics, since RSVD performance and accuracy are closely tied to the encountered spectra of singular values. This question applies especially to critical systems where the singular values are expected to decay slowly.

In this paper, we demonstrate superior performance of RSVD in the very original application field of TN methods, namely in identifying ground-state properties of low-dimensional quantum lattice Hamiltonians. We confirm significant speedup in different physical scenarios, including situations when the system is critical. Embedded in full-fledged TN simulations, we compare the RSVD against the truncated full SVD from state-of-the-art LAPACK implementations D/ZGESDD [46], referred to as TSVD in the following. As benchmarks, we use variants of the quantum Ising model in imaginary TEBD time evolution and a DMRG-style groundstate search with the hierarchical binary tree TN (TTN) [16,4752]. It will become apparent that a simple replacement of TSVD with RSVD code can lead to speedups between one and two orders of magnitude, while preserving the same precision, even when state-of-the-art TN techniques are employed [23,24].

The paper is organized as follows: First, we explain the use of truncated SVD as a tool of information compression in typical TN scenarios in Sec. II. We continue with a short review of the RSVD method and how it can help achieve faster compression in Sec. IIB. We then introduce our benchmark models in Sec. III and present a detailed performance analysis by switching from TSVD to RSVD in Sec. IV. Section V concludes the paper with a discussion of the results and with practical tips for the implementation and identification of situations that may benefit from RSVD.

\section{LOW-RANK FACTORIZATION}

The maximal bond dimension $\chi$ of a TN is a fundamental parameter: It can be linked to the amount of quantum entanglement that can be hosted in the network state [6]. At the same time, $\chi$ determines the computational complexity of algorithms performed on the network. Typical operations include the computation of expectation values, propagation in real or imaginary time, and renormalization steps updating the network description in iterative algorithms. All these operations can result in the growth of index dimensions beyond the maximally allowed bond dimension. A compression step is then achieved by means of a truncated SVD.

\section{A. Truncated SVD}

Let $A$ be a real- or complex-valued $m$-by- $n$ matrix with $m \geqslant n$. In our case, $A$ usually represents the contraction of two tensors, and it can also be given in the form of a matrix product $X^{\prime} Y^{\prime}$. The compression step then provides a rank- $\chi$ factorization $X Y$ which is a good approximation $A \approx X Y$, but also limits $X$ to an $m$-by- $\chi$ matrix and $Y$ to a $\chi$-by- $n$ matrix. A standard solution is to compute the rank- $\chi$ truncated SVD as follows:

\author{
Algorithm TSVD \\ Input: $m$-by- $n$ matrix $A$, integer $\chi$ \\ Output: rank- $\chi$ truncated SVD of $A$ \\ 1 Compute the full SVD of $A=U \Sigma V^{\dagger}$ \\ 2 Extract the $\chi$ largest singular values from $\Sigma$ and corresponding \\ columns of $U$ and $V$
}

In particular, $U$ is an $m$-by- $n$ matrix, $\Sigma$ and $V$ are $n$-by- $n$ matrices, and we assume $\Sigma$ is the diagonal matrix containing the singular values $\sigma_{j}=\Sigma_{j j}$ in descending order $\sigma_{1} \geqslant \sigma_{2} \geqslant$ $\cdots \geqslant \sigma_{n} \geqslant 0$. We then discard the $n-\chi$ smallest singular values (assuming $\chi \leqslant n$ ) and obtain, for instance, $X_{i j}=U_{i j}$ and $Y_{j k}=\sigma_{j} V_{j k}^{\dagger}$ for $j=1, \ldots, \chi$.

The truncation error $\delta_{\text {trunc }}:=\|A-X Y\|$ is then known to be minimal $[53,54]$ when measured in spectral norm $\left(\delta_{\text {trunc }}=\right.$ $\left.\sigma_{\chi+1}\right)$ or Frobenius norm $\left(\delta_{\text {trunc }}^{2}=\sum_{k=\chi+1}^{n} \sigma_{k}^{2}\right)$.

The availability of highly optimized SVD routines makes the implementation of TSVD straightforward. However, while it provides high accuracy, actually computing all $n$ singular values and vectors in the full SVD of $A$ still requires $O\left(m n^{2}\right)$ floating-point operations.

When the compression ratio $n / \chi$ becomes large, a more efficient protocol for computing the truncated SVD of $A$ is the RSVD algorithm.

\section{B. Randomized algorithm}

The basic idea of RSVD is simple: First, the input matrix $A$ is approximated with a rank- $\ell$ matrix $A_{\ell} \approx A$, which is obtained with randomness. From there, a rank- $\chi$ truncated SVD of $A_{\ell}$ is obtained at significantly lowered computational cost compared to a full SVD of $A$.

Two characteristic choices lead to an accurate $A_{\ell}$ :

(1) oversampling the approximation with $\ell>\chi$ [55] and

(2) employing a randomized power iteration of length $q$ [56].

We state the complete algorithm first, as put forward in Ref. [44], and then discuss the impact of both parameters $\ell$ and $q$ on computational cost and quality of the outcome.

\author{
Algorithm RSVD \\ Input: $m$-by- $n$ matrix $A$, integers $\chi, \ell, q$ \\ Output: approximate rank- $\chi$ truncated SVD of $A$ \\ 1 Generate an $n$-by- $\ell$ Gaussian matrix $\Omega$ \\ 2 Compute $Y:=\left(A A^{\dagger}\right)^{q} A \Omega$ \\ 3 Store in $Q$ the orthonormalized columns of $Y$ \\ 4 Compute the rank- $\chi$ truncated SVD of $B:=Q^{\dagger} A$
}

In detail, the algorithm begins by drawing a random test matrix $\Omega$ from a standard Gaussian distribution in step 1 . Note that other choices may work as well and that the quality of random numbers is not of crucial importance. Step 2 then produces an $m$-by- $\ell$ sample $Y$ of the range of $A$, by multiplying the columns of the test matrix with $\left(A A^{\dagger}\right)^{q} A$. 
This process emphasizes the most relevant singular vectors, associated with large singular values $\sigma$, by a factor of $\sigma^{2 q+1}$. In order to maintain numerical stability when these factors range over several orders of magnitude, step 2 is carried out as a power iteration with subsequent $\mathrm{QR}$ factorizations to keep the sample orthonormal (see Ref. [44], Algorithm 4.4). Step 3 then provides a basis of the sampled, relevant contributions to the range of $A$ in the orthonormal columns of the $m$-by- $\ell$ matrix $Q$. A rank- $\ell$ approximation of $A$ is now available by projection into that subspace: $A_{\ell}:=Q Q^{\dagger} A$. Such an explicit construction is, however, not required. Instead, step 4 invokes a rank- $\chi$ TSVD factorization $\tilde{U} \tilde{\Sigma} \tilde{V}^{\dagger}$ of the typically much smaller $\ell$-by- $n$ matrix $B:=Q^{\dagger} A$. Due to $A_{\ell} \approx A$, the $\chi$ largest singular values of $A$ are approximated in $\tilde{\Sigma}$. If required, approximate associated left and right singular vectors of $A$ are given by $Q \tilde{U}$ and $\tilde{V}$, respectively. Both are exact isometries.

The average RSVD compression error $\varepsilon_{\text {RSVD }}:=$ $\mathbb{E}\left(\left\|A-Q \tilde{U} \tilde{\Sigma} \tilde{V}^{\dagger}\right\|\right)$ depends on the spectrum of singular values and can be made arbitrarily close to the minimal truncation error $\delta_{\text {trunc }}$ in either Frobenius or spectral norm: Following the analysis in Ref. [57], a minimal oversampling of $\ell \geqslant x+2$ already guarantees

$$
\varepsilon_{\mathrm{RSVD}} \leqslant \sqrt{\delta_{\text {trunc }}^{2}+\mathcal{C}^{2}(n, \ell) \chi \sigma_{\ell-1}^{2}\left(\sigma_{\ell-1} / \sigma_{\chi}\right)^{4 q}}
$$

where $\mathcal{C}^{2}(n, \ell)=(4 e / 3)^{2}(\sqrt{n-\ell+2}+\sqrt{\ell}+7)^{2} \ell(e$ being Euler's number) is of complexity $O(n \ell)$, i.e., bounded by a bilinear growth in $n$ and $\ell$ from above, with $\ell \leqslant n$. Note that $\delta_{\text {trunc }}$ depends on the selected norm, unlike the additional terms introduced by the randomized approach. While highest accuracy is expected for quickly decreasing singular values, a striking feature of RSVD is that already small powers $q>0$ drive those contributions, which add to $\delta_{\text {trunc }}$ in Eq. (1), to zero exponentially fast, even in cases of slowly decaying singular values. Furthermore, sufficient oversampling in $\ell$ makes the probability of a substantial deviation from the average error bound arbitrarily small [57].

Throughout our benchmarks, we make the conservative choice $\ell=2 \chi$, which is suitable to keep the RSVD error within a small factor of $\delta_{\text {trunc }}$ even for $q=0$ [44]. In this configuration, RSVD promises an asymptotic speedup over TSVD in the order of the compression ratio

$$
T_{T} / T_{R} \sim n / \chi,
$$

where $T_{T}$ and $T_{R}$ are the times required by TSVD and RSVD to decompose the same input matrix $A$. The proportionality is due to the lower RSVD computational complexity, which is dominated by the matrix-matrix products of $O(m n \ell(q+1))$ in sampling $Y$.

The improved scaling of the RSVD algorithm is complemented by its conceptual simplicity, which directly translates to a fast, stable, and easily parallelizable implementation in terms of highly optimized linear algebra routines as provided by level-3 BLAS and LAPACK [46]. Various RSVD implementations are available, for instance in MATLAB [58], in R [59], and via $\mathrm{C}$ libraries such as RSVDPACK [60] or RRSVD-RACKAGE [45], which our benchmarks are based on.

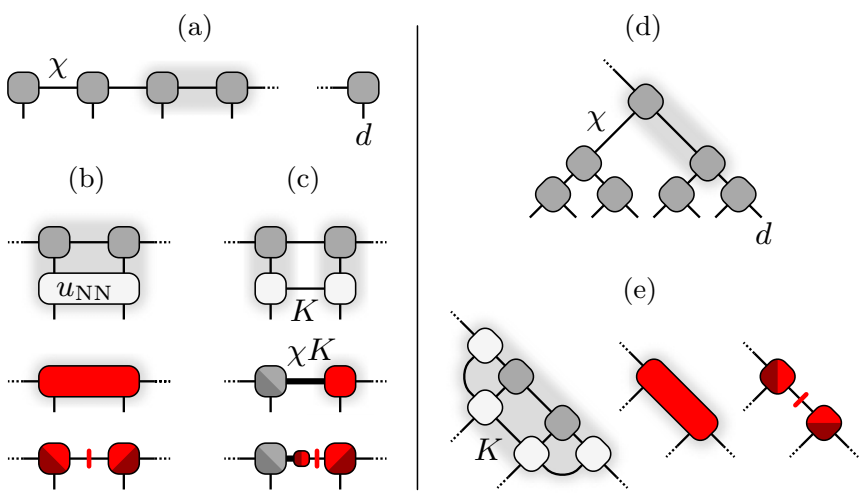

FIG. 1. Compression steps in the benchmarked tensor networks of bond dimension $\chi$ and local dimension $d$. The truncated SVDs (highlighted in red) retain at most $\chi$ largest singular values (red bar) and produce isometric tensors (shaded). (a) Diagrammatic representation of the MPS used in TEBD. (b) Absorbtion of the nearest-neighbor evolution exponential in form of a four-link tensor "block" (top to bottom). (c) Absorbtion of a sum over $K$ Kronecker products results in a different compression problem. (d) Binary tree TN ansatz for variational energy minimization. (e) Two-site update: The energy of an effective Hamiltonian (left) is minimized by optimizing adjacent tensors in form of a four-link matrix which is then rank- $\chi$ factorized (right).

\section{BENCHMARKS}

We benchmark RSVD against TSVD performance, when employed in state-of-the-art TN algorithms. Our focus lies on closed-system ground states, and we compare both run time and precision of the relevant physical quantities in the outcomes.

We first outline the $\mathrm{TN}$ algorithms that drive our benchmark simulations and the role played by compression. Afterward, we report model Hamiltonians and parameters. We close this section with a brief account on the numerical implementation.

\section{A. TN algorithms}

We employed TEBD imaginary time evolution on MPSs and DMRG-style variational ground-state search in the TTN [51] with double-tensor optimization. Both algorithms are well-established techniques in ground-state search of quantum lattice Hamiltonians. They iteratively approximate those ground states in TNs of a selected maximal bond dimension $\chi$, defined over $d$-dimensional "physical" tensor indices that correspond to lattice sites [see Figs. 1(a) and 1(d)]. Specifically, both algorithms perform local update steps on adjacent tensors, which require a truncated SVD to recompress bond indices. Note that it is the absence of loops (network cycles) in MPS and TTN geometries that makes truncated SVD an optimal protocol here, as it maintains maximum quantum fidelity between the states before and after the compression of a single bond $[3,4,61]$.

The two methods, however, rely on different local update steps:

In the TEBD algorithm, designed for time evolution with nearest-neighbor interactions, the update step consists of an application of a (real or imaginary) time-evolution exponential 
on two adjacent lattice sites. In standard TEBD, the exponential takes the form of a single four-index tensor or "block" (B) $u_{\mathrm{NN}}$. It can also be given by a sum of Kronecker products (P) of single-site operators $\sum_{k=1}^{K} u_{L}^{k} \otimes u_{R}^{k}$, which can be more time and memory efficient for $K<d$. Both strategies pose different compression problems [Figs. 1(b) and 1(c)]: The block update contracts directly into a square matrix of dimension $\chi d$, while in the product update we obtain a $(\chi K)$-by- $(\chi d)$ matrix instead. In both cases, the resulting matrix $A$ must be compressed into a rank- $\chi$ factorization with compression ratios $d$ and $\min (K, d)$, respectively. Consequently, we expect RSVD to significantly speed up TEBD simulations on lattices with larger local dimensions $d$ : In terms of computational complexity, TEBD with typical bond dimension $\chi \geqslant d$ is dominated by the TSVD compression step of $O\left(\chi^{3} d^{3}\right)$ in block (B) and $O\left(\chi^{3} K^{2} d\right)$ in product (P) updates for $K \leqslant d$. The asymptotic RSVD speedup can reduce this scaling to $O\left(\chi^{3} d^{2}\right)$ (for B), as demonstrated in Ref. [45], and to $O\left(\chi^{3} K d\right.$ ) (for $\mathrm{P})$, respectively - which are typical costs exhibited by other operations within TEBD as well.

In the TTN setting, instead, the update step directly replaces two adjacent tensors with a matrix $A$ associated to the lowest eigenvector of an effective Hamiltonian. The matrix $A$ is at most a $\chi^{2}$-by- $\chi^{2}$ square matrix. On some lower levels of the tree geometry, smaller dimensions can be encountered, with $d^{2}$ at the physical indices on the bottom [Fig. 1(e)]. The majority of run time, however, is spent on the large update matrices, and these require a compression by a ratio $\chi$. A massive speedup of the compression step, in the order of the bond dimension, can thus be expected from employing RSVD instead of TSVD.

A feature of all simulations is that we explicitly target the symmetry-invariant ground states under certain global Abelian symmetries of the Hamiltonian. These grant us an inner block structure in all tensors, which enhances efficiency and precision of the simulation $[23,24]$. In the compression problem, we therefore encounter strictly block-diagonal matrices $A$, encoded in $N$ nontrivial blocks. The dimensions of these blocks correspond to degeneracies of symmetry sectors, and add up to the respective full dimensions of $A$. In all benchmarked situations, $N$ equals the (small) number of global symmetry sectors, and the optimal TN ground-state approximations display more or less evenly sized block dimensions. Since matrix factorizations can be done blockwise, all the actual matrix dimensions passed to the truncated SVD algorithm are thus roughly those of $A$ divided by $N$. But as the truncation rank $\chi_{s} \approx \chi / N$ per block is similarly reduced, no change in the compression ratio and hence in the asymptotic speedup occurs.

Note that the truncation rank per block is usually not known a priori, as it depends on the number of large singular values $\sigma_{j}>\sigma_{\chi}$ therein. This information is only directly available with TSVD, where all singular values of all blocks are computed. RSVD, on the other hand, delivers just the requested number of singular values for each block, and some estimate of the appropriate truncation $\chi_{s}^{\prime} \approx \chi_{s}$ must be made beforehand. After RSVDs are then performed in all sectors, we postselect the $\chi$ largest singular values and obtain the new optimal block dimensions $\chi_{s}$. In our TEBD simulations, we choose a blockwise truncation rank $\chi_{s}^{\prime}=\chi / N+c$ with a small constant $c$ that allows for some variation in sector sizes (typically less than 5\%). For TTN, we instead make the simplest maximal choice $\chi_{s}^{\prime}=\chi$, which reduces the achievable speedup by a (small) factor $N$ but does not require any estimates.

\section{B. Models}

We simulated the quantum Ising model with ferromagnetic interaction in a tunable transverse field $h$, on two different lattices: First, a 1D spin- $S$ chain of length $L$ with Hamiltonian

$$
H_{\text {chain }}=-\frac{1}{S^{2}} \sum_{j} X_{j} X_{j+1}+\frac{h}{S} \sum_{j} Z_{j}
$$

where $X$ and $Z$ are local spin operators (we set $\hbar=1$ ) and subscripts denote application sites. In general, in a computational spin- $Z$ eigenbasis $\{|m\rangle\}$ of local dimension $d=2 S+1$ with integer or half-integer magnetic quantum numbers $m \in$ $\{-S,-S+1, \ldots, S\}$, we have

$$
\begin{aligned}
\left\langle m^{\prime}|Z| m\right\rangle= & m \delta_{m^{\prime}, m}, \\
\left\langle m^{\prime}|X| m\right\rangle= & \sqrt{(S+1)\left(m+m^{\prime}-1\right)-m m^{\prime}} \\
& \times\left(\delta_{m^{\prime}, m+1}+\delta_{m^{\prime}+1, m}\right) / 2 .
\end{aligned}
$$

For $S=1 / 2, X$ and $Z$ reduce to standard Pauli matrices and the model is exactly solvable with quantum critical point at $|h|=h_{c}=1$. For $S \rightarrow \infty$, the transition point shifts with $h_{c} \rightarrow 2$ [62]. The TEBD is performed for $S>1 / 2$ in open boundary conditions with values of $h$ in various distances to the critical points, which we estimated from finite-size scaling techniques [63]. In our TTN benchmark, we focus exclusively on $S=1 / 2, h=h_{c}$ in periodic boundary conditions.

The second benchmark is the simulation of a spin- $1 / 2$ two-dimensional (2D) square-lattice Ising model in cylindrical boundary conditions of length $L$ and circumference (or width) $W$. With respective site subscripts $i$ and $j$, the Hamiltonian reads

$$
H_{\mathrm{cyl}}=-\sum_{i, j} X_{i, j} X_{i, j+1}-\sum_{i, j} X_{i, j} X_{i+1, j}+h \sum_{i, j} Z_{i, j} .
$$

By summation over $i$, we map this Hamiltonian onto an open chain of length $L$ with local dimension $d=2^{W}$. For reasonably small values $W$, the ground state can be approximated in a MPS and its critical behavior can be studied with DMRG [64]. We performed imaginary TEBD at various values of $h$, including points in proximity of the critical field at around $h_{c} \approx 3.044$, as reported with high precision in Monte Carlo and TN studies on the square lattice $[65,66]$.

As is well known, in the thermodynamic limit, the oneand two-dimensional Ising models of Eqs. (3) and (5) exhibit spontaneous ferromagnetic order for $|h|<h_{c}$, which breaks down in the paramagnetic phase for $|h|>h_{c}$. Both phases are gapped; however, at $|h|=h_{c}$, the systems become critical and gapless.

In the case of $1 \mathrm{D}$ lattices, we know that the ground states of a short-ranged, gapped system obey area laws for the entanglement entropy, while this is not true for a critical, gapless system [7-10]. Since squares of the singular values in loop-free $\mathrm{TN}$ compression steps correspond to reduced density eigenvalues of lattice bipartitions, singular values are directly linked to bipartite entanglement measures such as the von 
Neumann entropy, and thus the error analysis Eq. (1) of RSVD is linked to the physical properties of the ground state. For this reason, we perform our benchmarks at various values of $h$, including values in close proximity to $h_{c}$. We expect the latter to pose the most demanding situation for RSVD due to a potentially slow decay of tail singular values [67], which make greater amounts of computational resources necessary (via parameters $q, \ell$ ) to avoid larger errors in Eq. (1). As a comment, we remark that the benchmarked MPS and TTN simulations are best suited for noncritical systems due to finite bond dimensions $\chi$ that limit correlations and entanglement. However, the selected finite lattice sizes admit simulations at and around $h=h_{c}$, as is typical in extrapolating critical properties via finite-size scaling techniques $[63,68]$. Furthermore, TTN have capabilities beyond MPS in encoding quantum critical ground states [16].

Both Ising models in Eqs. (3) and (5) exhibit a global parity symmetry because their Hamiltonians commute with $\bigotimes_{j=1}^{L} P_{j}$, being defined locally by $\left\langle m^{\prime}|P| m\right\rangle=(-1)^{m+S} \delta_{m^{\prime}, m}$. Local basis states transform as $P\left|m^{ \pm}\right\rangle= \pm\left|m^{ \pm}\right\rangle$and fall either in the even "+" or odd sector "-_" of dimensions $d_{+}$, $d_{-} \approx d / 2$ respectively. Rotations in the cylindrical boundary conditions provide an additional Abelian $Z_{W}$ cyclic symmetry for (5). As a consequence, even and odd sectors further decompose into $W$ different angular momentum sectors. As mentioned in Sec. III A, we encode these symmetries explicitly, which allows us to restrict the TN state representation to the ground-state global invariant sector $s=0$, that is, the even-parity and rotationally invariant subspace.

\section{Implementation}

Here we report the detailed implementation of a fair run time and precision comparison between TSVD and RSVD, and discuss technical details of the benchmarks.

We performed complete runs of our TEBD and TTN benchmark algorithms by iterating double-tensor updates until the energy expectation value of the TN state stagnates within some threshold $\delta E$. Each run was repeated for different field $h$, maximal bond dimension $\chi$, lattice length $L$, and a selected spin $S$ or width $W$, either with TSVD or RSVD in the compression steps.

For the precision comparison, we extracted expectations of energy and magnetization order, correlation and entanglement properties, and singular values from the produced final states. The magnetization order $M$ was measured from nonlocal correlations,

$$
M=\sqrt{\sum_{k \neq k^{\prime}}\left\langle X_{k} X_{k^{\prime}}\right\rangle / \mathcal{N}},
$$

where $k$ goes over all lattice sites and $\mathcal{N}$ counts the number of expectations summed over. The estimate for the correlation length $\bar{\xi}$ was computed from expectations values of $X_{(k)} \equiv X_{k}$ in the chain and $X_{(k)} \equiv X_{i, j}$ in the cylinder as follows:

$$
\bar{\xi}=\sqrt{\frac{\sum_{r>0}(r-1)^{2} C_{r}}{2 \sum_{r>0} C_{r}}} .
$$

Here, $C_{r}$ denotes the bulk average over MPS sites $j$ of $\left\langle X_{(i,) j}, X_{(i,) j+r}\right\rangle$. The additional site index $i$ appears only in the two-dimensional model and is averaged over as well to extract only the horizontal correlation length subject to compression through the MPS bond links. Note that $\bar{\xi}$ tends to underestimate the actual correlation length and saturates below $L / \sqrt{6}$ if it becomes large compared to the system size. Furthermore, profiles of the von Neumann entropy $S_{N}(j)=-\sum_{k} \lambda_{k}^{2} \log \left(\lambda_{k}^{2}\right)$ have been obtained from the compressed singular values at MPS bonds $j=1, \ldots, L-1$.

We also profiled the individual run times spent in the truncated SVDs of compression steps, $T_{T}$ and $T_{R}$, and the time spent in all remaining parts of the algorithm, $\bar{T}_{T}$ and $\bar{T}_{R}$, for TSVD and RSVD runs, respectively. All these run times have been divided by the number of iterations performed in the simulation. However, we have found no substantial differences in the number of update steps performed with TSVD and RSVD, as reported in Sec. IV. We therefore obtain the average speedup in compression due to RSVD from

$$
\tau:=f \times T_{T} / T_{R},
$$

where $f:=\bar{T}_{R} / \bar{T}_{T}$ is the ratio of run times spent outside compression. Since our benchmarks have been performed on shared cluster nodes, we introduced the factor $f$ to equalize the effect of the computational environment on the bare compression times. Thus, simulation runs that were slowed done by other computations on a cluster node can be fairly compared to faster executed simulation runs.

The complete simulation protocol for TEBD was as follows: Starting from a product state with randomized tensors of bond dimension one, the algorithm is run in imaginary time with some sufficiently large initial time step $d t$ in the local imaginary time evolution exponential. After a few first iterations out of typically many hundred, the bond dimension saturates the allowed maximum, and we can safely assume $\chi$ to be the typical compression rank. The simulation stops when convergence of the energy is detected as follows: Throughout the simulation, the change of the expectation value of the energy is monitored in regular intervals. Whenever this change drops below the targeted precision threshold $\delta E$, the simulation time step $d t$ is subsequently reduced by a constant factor. Convergence is declared when the total energy decrement between two time-step reductions falls below $\delta E$, too. With smaller choices of $\delta E$, better approximations of the final MPS to the actual ground state of the system can be expected within the bond dimension $\chi$, but at the cost of increased number of iterations and run time.

The TTN ground-state search employs randomized initial states remaining at maximal bond dimension throughout the entire simulation. The same initial states were used in comparative TSVD and RSVD runs. The algorithm then performs sequences of double-tensor updates on adjacent tensors, until the difference in energy expectation between subsequent sweeps falls below machine precision.

All simulations were carried out in double precision arithmetic with complex numbers, except for the imaginary TEBD on the spin- $1 / 2$ chain which we benchmarked in a TN representation with real elements, a common choice to enhance efficiency under time-reversal invariance. Linear algebra computations (BLAS, LAPACK) where performed with 
(a)

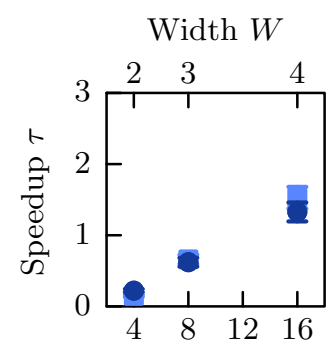

Local dimension $d$

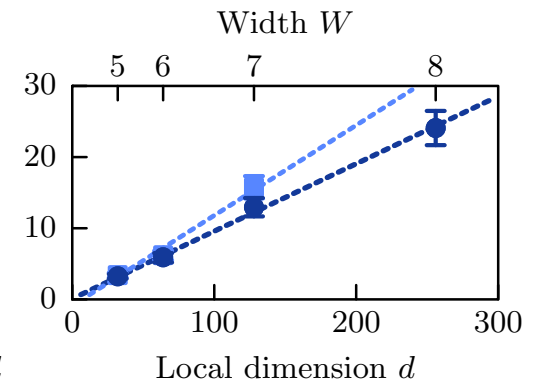

(b)

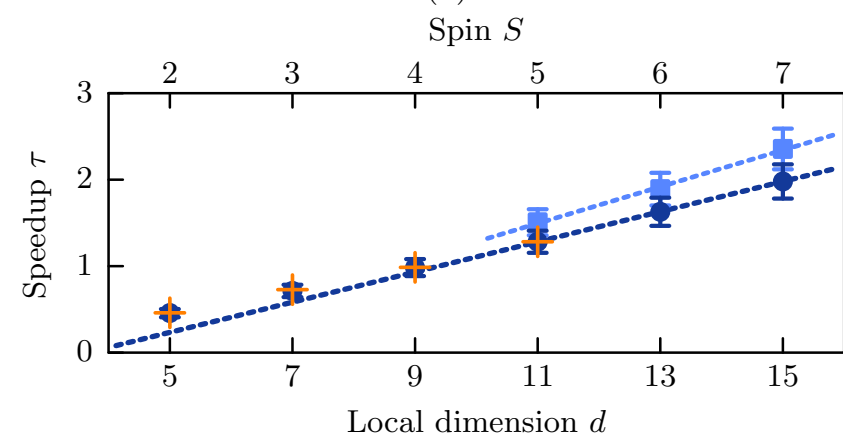

FIG. 2. Speedup in compression step due to RSVD in TEBD simulations of increasing local dimensions. (a) 2D Ising model ( $L=$ $30)$ as a function of the width $W$. (b) $1 \mathrm{D}$ Ising model $(L=100)$ as a function of local spin $S$. Dark and light blue points represent data at bond dimensions $\chi=100$ and $\chi=150$, respectively, both from block update and fixed convergence criteria (1D: $\delta E=10^{-13}, 2 \mathrm{D}$ : $\delta E=10^{-8}$ except $W=8$ was stopped before convergence). Error bars indicate a $10 \%$ error estimate in speedups. Orange crosses show speedup in higher precision target $\delta E=10^{-14}, \chi=100$. Dashed lines are linear fits for $d>10$ with slopes $\approx 0.10,0.13$ (2D) and $0.18,0.21$ (1D) for $\chi=100,150$ respectively.

the INTEL MATH KERNEL LIBRARY (MKL) in versions 11.x. Our fully truncated TSVD implementation is based on the LAPACK D/ZGESDD divide-and-conquer algorithm. For RSVD, we employed the fixed-rank implementation from the RRSVD package [45] with parameters $q=4, \ell=2 \chi$ (see Sec. II B) for any targeted truncation rank $\chi$. This implementation employs LAPACK D/ZGESVD for the final factorization in step 4 of the RSVD algorithm. All simulations were executed with singlethreaded compression step on 16-way Intel Xeon E5-2670 (2.6-GHZ) compute nodes.

\section{RESULTS}

We first report the speedups obtained from upgrading compression steps from TSVD to RSVD. We then present evidence that no loss of precision occurs due to RSVD. Finally, we present selected ground-state properties and spectra of singular values that we encountered in our benchmarks.

All the following speedups have been obtained from independent simulations according to Eq. (8) with an estimated uncertainty of at most $\Delta \tau \approx 10 \%$. Equal numbers of RSVD and TSVD compression steps were performed in all TTN simulations. Some imaginary TEBD runs converged in less (a)

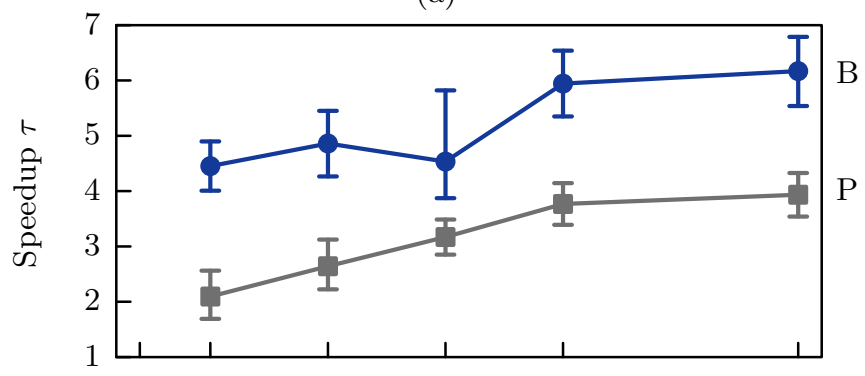

(b)

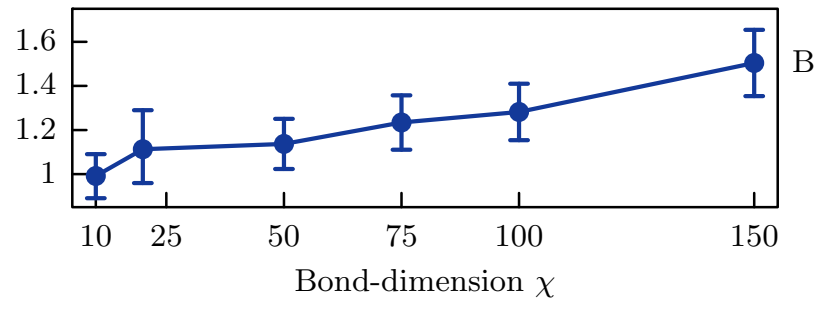

FIG. 3. Dependency of RSVD speedup on the bond dimension in TEBD simulations. (a) 2D Ising model, speedup at width $W=6$, $L=30$, and $\delta E=10^{-8}$. Blue and gray: block (B) and product (P) updates, respectively. (b) $1 \mathrm{D}$ Ising model, speedup at spin $S=5$, $L=100$ and $\delta E=10^{-13}$. Both panels share the same $x$ axis.

iterations with either RSVD or TSVD, but those fluctuations were negligible compared to $\Delta \tau$.

Speedups up to $\tau \approx 24$ have been reached in TEBD simulations of increasing local dimensions, as shown in Fig. 2 for the one- and two-dimensional Ising models of Eqs. (3) and (5). We observe that RSVD outperforms TSVD for $d>10$, with speedups directly proportional to $d$ as predicted by the asymptotic cost analysis in Sec. III A. These speedups remain stable under different algorithm parameters, such as changes in convergence criteria [orange crosses in Fig. 2(b)]. We also found no significant dependency on the transverse field $h$ : Thus, all speedups are geometric means over five (2D) and ten (1D) different values of $h$ in various distances from (including close proximity to) the critical point, and each speedup falls within the error bars.

In all cases, however, the speedup tends to increase with the bond dimension, as shown in Fig. 3 for selected one- and two-dimensional TEBD simulations. The latter suggests some saturation at high bond dimension. Again, all speedups shown are geometric means over at least ten simulations at transverse fields $h$ in various distances from $h_{c}$, which had no significant impact on the speedup, as can be seen from the error bars that always enclose minimal and maximal speedup.

Complementary to our TEBD results, the TTN benchmarks demonstrate massive RSVD speedups already for spin $1 / 2$, when bond dimensions are scaled up: For instance, at $\chi=60$ we found $\tau \approx 6$, while $\chi=100$ already provided us with $\tau \approx$ 11 , both on a lattice of length $L=64$.

Next, we assess the accuracy of the final states delivered by our TSVD and RSVD benchmarks. To this end, we compare the simulation errors in energy expectation value $E$ and nonlocal magnetization order parameter $M$ of Eq. (6) for various simulation parameters such as $h, \chi$, and precision target 
(a)

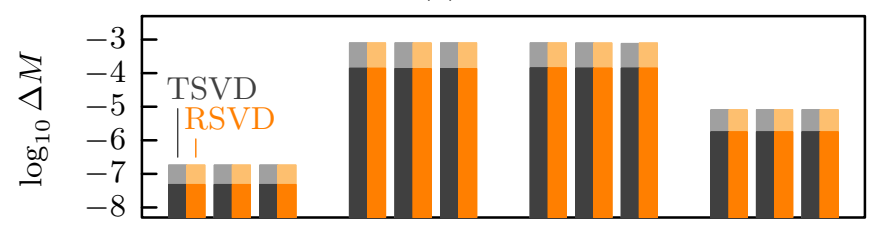

(b)

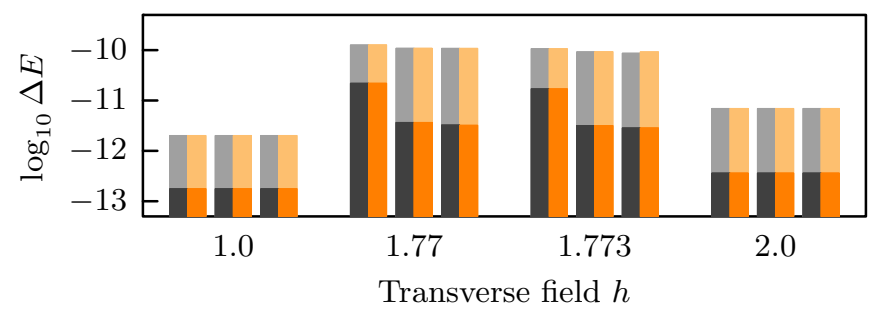

FIG. 4. Relative errors in 1D TEBD simulations with TSVD and RSVD. (a) Error in final-state energy $\Delta E$. (b) Error in magnetization $\Delta M$. Each group of three bars shows the error with increasing bond dimensions $\chi=50,75,100$ (left to right) at a given transverse field (both panels share the same $x$ axis). Black (orange) bars correspond to TSVD (RSVD) results at convergence thresholds $\delta E=10^{-11}$ (light shaded) and $10^{-13}$. All errors have been obtained from extrapolated ground state values for $S=5, L=100$ in the ferro- $(h=1.0)$ and paramagnetic $(h=2.0)$ phases as well as close to the critical point.

$\delta E$. The errors are computed from differences $\Delta E=(E-$ $\left.E_{\text {best }}\right) / E_{\text {best }}$ and $\Delta M=\left|M-M_{\text {best }}\right| / M_{\text {best }}$ to high-precision data $E_{\text {best }}$ and $M_{\text {best }}$, respectively. In TEBD simulations, $E_{\text {best }}$ and $M_{\text {best }}$ have been extrapolated from bond dimensions and precisions up to $\chi=150, \delta E=10^{-14}$ using TSVD, with uncertainty smaller than all observed differences $\Delta E$ and $\Delta M$ (typically one or more orders of magnitude). We found that both TSVD and RSVD produce comparable simulation errors in all benchmarks, as exemplified in Fig. 4 for TEBD simulations of the one-dimensional Ising model for $L=100$ and $S=5$. We found similar results for up to $L=400$ in various precision targets and bond dimensions $\chi \leqslant 100$ in both para- and ferromagnetic phases as well as close to the critical point. In two-dimensional TEBD simulations at $W=6, L=$ 30 and in the TTN benchmarks, TSVD and RSVD results even matched within computational precision.

The range of physical properties covered by our benchmarks is demonstrated in Fig. 5, where the upper panels [Figs. 5(a) and 5(b)] show the magnetization $M$ and the estimate for the correlation length $\bar{\xi}$ [see Eq. (7)] in the final TEBD simulation states. These results, taken from TSVD runs of 1D and 2D Ising models for some of the benchmarked transverse fields $h$, display values of magnetic order and correlation lengths spanning the entire spectrum of possible outcomes. Furthermore, the von Neumann entropies $S_{N}(j)$ on the MPS bonds confirm area laws in both ordered and unordered phases as well as typical corrections near the 1D critical point, which are well described by a fit to $S_{N}^{C}(j)=a+c / 6 \log \{L / \pi \sin (\pi j / L)\}$ with some constants $a, c$ [69]. The corresponding singular values are detailed in the bottom panels [Figs. 5(c) and 5(d)]. Within the bond dimensions $\chi_{s}$ of individual symmetry sectors, they are well fitted by power-law decays $\sigma_{k} \approx\left(C_{1} k+C_{2}\right)^{-\gamma}$ (a)

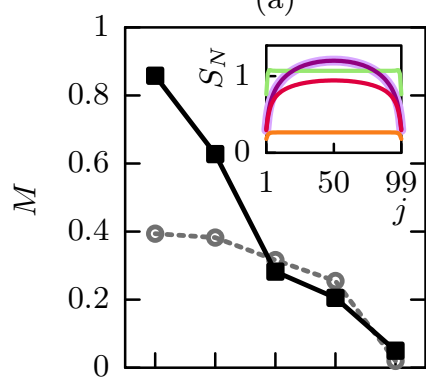

(c)

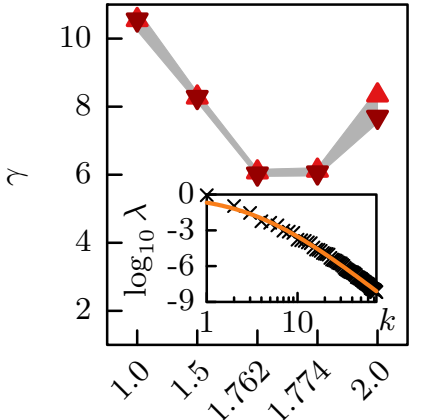

Transverse field $h$ (b)

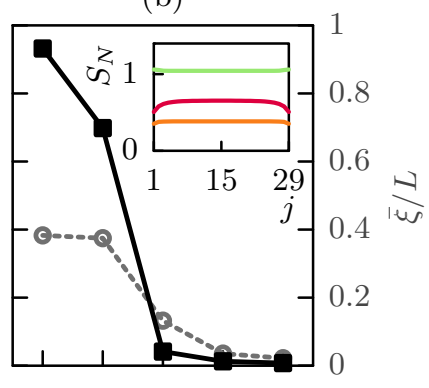

(d)

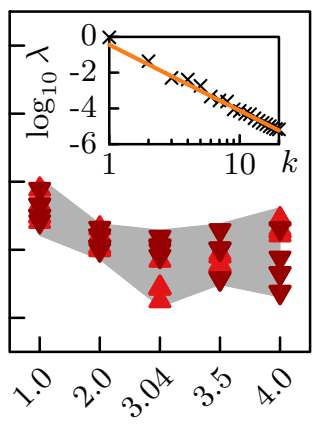

Transverse field $h$
FIG. 5. Correlation properties and singular values in TEBD simulated ground states in various transverse fields $h$, for the 1D Ising model at $S=5, L=100$ [left panels (a), (c)] and the 2D Ising model at $W=6, L=30$ [right panels (b), (d)]. Panels (a) and (b): Magnetization $M$ (black) and estimates for the correlation length $\bar{\xi} / L$ (gray dashed). Errors are smaller than point sizes. Insets show von Neumann entropies $S_{N}(j)$ from singular values on MPS bonds. Inset (a): $h=1.5$ (green), 1.762 (purple with light-purple fit $S_{N}^{C}(j)$, see text), 1.774 (red), 2.0 (orange). Inset (b): $h=2.0$ (green), 3.04 (red), 3.5 (orange). Panels (c) and (d): Decay exponents $\gamma$ of singular values. Upward (downward) pointing triangles indicate even (odd) sectors, respectively. Shaded area encloses fit errors. Insets show singular values (black) at a central bond in the invariant sector for near-critical fields and a polynomial fit $\sigma_{k} \approx\left(C_{1} k+C_{2}\right)^{-\gamma}$ (orange, $C_{2}=0$ in 2D). Inset (c): $h=1.7735$. Inset (d): $h=3.04$. Panels share $x(y)$ axes.

with fit constants $C_{1}, C_{2}$, and decay exponents $\gamma$ ranging from -2 to -11 .

This decay of singular values, which relates physical properties to RSVD performace (as discussed in Sec. III) is further analyzed in Fig. 6 where we present complete spectra of singular values from the local compression problems $A$, including the truncated tail of small singular values, for a central bond and critical transverse field. In both TEBD [Fig. 6(a)] and TTN [Fig. 6(b)] simulations, the spectrum of singular values $\lambda_{k}$ can be separated into two parts: For $k \leqslant \chi_{s}$, the spectrum appears to undergo only minor changes throughout the algorithm run time and is well described by the actual decay in the final (ground) state (see Fig. 5 for TEBD) over the majority of the run time. For $k>\chi_{s}$, on the other hand, we observe a tail spectrum that does not necessarily follow the characteristics expected from the actual ground state (i.e., $\chi \rightarrow \infty)$. Namely, it changes significantly over the algorithm run time and exhibits the fastest decay in the final iteration(s) of the algorithm: In the case of TEBD, the tail can be seen to 
(a)
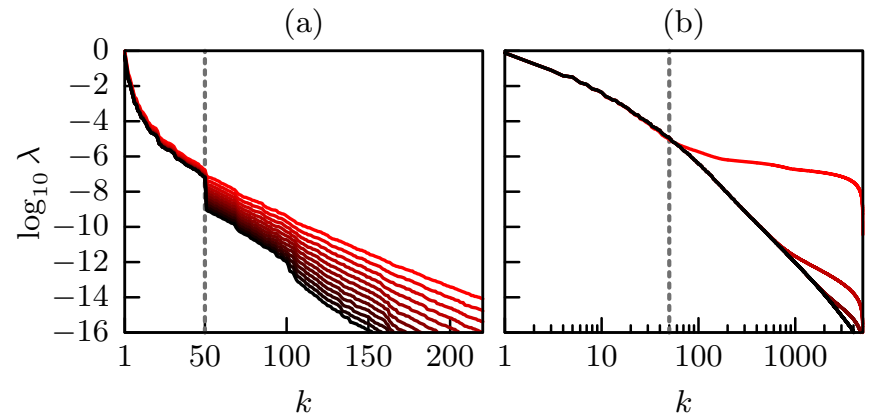

FIG. 6. Singular values $\lambda_{k}$ monitored over algorithm runtime in 1D Ising models at, or close to, the critical field. Both panels show values in the invariant sector at a central lattice bipartition with $\chi=100$. The dashed lines indicate the truncation at $\chi_{0}=50$. (a) Imaginary TEBD $\left(S=5, L=100, h=1.7735, \delta E=10^{-13}\right.$, block update B). The time step $d t$ was subsequently reduced to $d t_{i}=0.4 \times 0.7^{i}$ for $i=0, \ldots, 11$ (red to black). (b) TTN groundstate search $(S=1 / 2, L=128, h=1.0)$, after $i=0, \ldots, 4$ network updates (red to black).

be bounded by a rapid polynomial decay, well separated from the retained singular values as it finally becomes proportional to a very small evolution time step $d t$. In TTN, compression starts from a rather flat tail spectrum that quickly approaches an exponential decay. This demonstrates that the compression problem within the TN approximation becomes increasingly well conditioned for RSVD, even close to the phase transition, as the algorithm converges closer to the ground state. This allows RSVD to deliver higher precision [cf. Eq. (1), due to oversampling] with higher reliability right in the final stages of the algorithms when most needed.

\section{DISCUSSION AND OUTLOOK}

We provided evidence for substantially accelerated compression of tensor networks in all benchmarked algorithms by simply replacing the full truncated TSVD with the RSVD algorithm. In particular, RSVD outperformed TSVD with the expected asymptotic speedup, that is proportional to the compression ratio, when not more than $10 \%$ of singular values were retained.

Remarkably enough, we attained those speedups without loss of precision in the simulated ground states: With RSVD we reproduced local expectation values such as the energy, as well as long-range correlation and entanglement properties, with differences to TSVD simulations far smaller than the inherent ansatz errors due to a finite bond dimension or number of iterations performed. By benchmarking with encoded Abelian symmetries, we confirmed the RSVD speedup in reduced bond and local dimensions per sector. Even though small matrix sizes can reduce speedups, RSVD becomes increasingly useful with the typically large bond dimensions that are required for ground-state approximation. All results, moreover, hold up independently from the various physical scenarios, i.e., off and at quantum critical points over a wide range of correlation lengths and respective spectra of the singular values. The iterative nature shared by many $\mathrm{TN}$ algorithms has been observed to work in favor of RSVD in that the truncated tail singular values decayed quickly in the relevant final iterations, even close to phase transitions.

We expect the presented results to be robust and reproducible in a wide range of tensor network applications. For instance, our choice of RSVD parameters $(q, \ell)$ has been extremely conservative, as confirmed by the small differences to TSVD in the outcomes, and can be fine-tuned for much higher efficiency: Namely, by reducing $q$, RSVD might outperform TSVD for compression ratios as moderate as five or less. With RSVD, precision and efficiency of the compression can further be balanced dynamically, which promises significant reduction of run time in the earlier algorithm stages, as is already standard practice, for instance, in the eigensolver optimization steps in DMRG. In this regard, it may prove specifically useful that RSVD can also deliver a fixed error (instead of fixed rank) approximation: Parameters such as $\chi, \ell$, and possibly $q$ are then dynamically adjusted to deliver a compression within a given error bound [44,57]. Such dynamics might also provide an alternative route to fix the compressed sector sizes $\chi_{s}$ in the presence of symmetric TN, even though good estimates (for instance based on previous iterations) plus added oversampling work well as demonstrated. Moreover, ongoing development of the RSVD method itself may lead to further optimizations, such as modified power iteration schemes for faster convergence [70] or single-view algorithms [71].

With the benchmarked ground-state simulations, it is clear that RSVD is indeed not limited to open system real-time dynamics with TEBD [45], and we foresee a broad impact on DMRG and imaginary or real time evolution codes that operate on ground states, including short-time quenches [72] out of equilibrium via TEBD or the time-dependent variational principle [73]. This in turn could open new possibilities, for instance, in the numerical study of the Kibble-Zurek mechanism [74,75]. More generally, RSVD has great potential in all TN algorithms that make extensive use of truncated SVD with high compression ratios. This includes the various double-tensor update strategies that are regularly employed in DMRG and time evolution codes when Abelian or non-Abelian symmetries are encoded, and to avoid metastabilities that hinder convergence [76,77]. Another particularly promising scenario is the efficient approximation of environments in higher dimensional lattices, especially with PEPS in infinite 2D lattices. Prominent examples are iPEPS [78] by means of infinite TEBD [79,80], coarse-graining renormalization approaches [34,81,82], and corner transfer matrix methods based on CTMRG $[33,83]$ with potentially large compression ratios when bond dimensions are pushed up. Conceivable use cases for RSVD include, for example, lattice models with large local dimensions, lattices in higher dimensions, infinite lattices, and applications of TNs in quantum chemistry.

Note added. S. Morita et al. [84] has recently reported a complexity reduction of the TRG algorithm achieved by means of RSVD.

\section{ACKNOWLEDGMENTS}

We thank M. Gerster for discussions and sharing his TTN code. The authors gratefully acknowledge support from the Carl-Zeiss-Stiftung via Nachwuchsförderprogramm, the 
state of Baden-Württemberg through bwHPC, the Italian HPC facility CINECA through the TEDDI project, and the German Research Foundation (DFG) through the TWITTER grants. S.M. gratefully acknowledges the support of the DFG via a Heisenberg fellowship. This work was supported by the ERC Synergy grant BioQ.
[1] U. Schollwöck, Ann. Phys. 326, 96 (2011).

[2] R. Orús, Ann. Phys. 349, 117 (2014).

[3] S. R. White, Phys. Rev. Lett. 69, 2863 (1992).

[4] S. R. White, Phys. Rev. B 48, 10345 (1993).

[5] S. Rommer and S. Östlund, Phys. Rev. B 55, 2164 (1997).

[6] J. Eisert, Model. Simul. 3, 520 (2013).

[7] K. Audenaert, J. Eisert, M. B. Plenio, and R. F. Werner, Phys. Rev. A 66, 042327 (2002).

[8] M. B. Plenio, J. Eisert, J. Dreißig, and M. Cramer, Phys. Rev. Lett. 94, 060503 (2005).

[9] J. Eisert, M. Cramer, and M. B. Plenio, Rev. Mod. Phys. 82, 277 (2010).

[10] M. M. Wolf, Phys. Rev. Lett. 96, 010404 (2006).

[11] F. Verstraete, M. M. Wolf, D. Perez-Garcia, and J. I. Cirac, Phys. Rev. Lett. 96, 220601 (2006).

[12] L. Tagliacozzo, G. Evenbly, and G. Vidal, Phys. Rev. B 80, 235127 (2009).

[13] M. Gerster, M. Rizzi, P. Silvi, M. Dalmonte, and S. Montangero, Phys. Rev. B 96, 195123 (2017).

[14] G. Vidal, Phys. Rev. Lett. 99, 220405 (2007).

[15] G. Vidal, Phys. Rev. Lett. 101, 110501 (2008).

[16] P. Silvi, V. Giovannetti, S. Montangero, M. Rizzi, J. I. Cirac, and R. Fazio, Phys. Rev. A 81, 062335 (2010).

[17] R. Orús, T.-C. Wei, O. Buerschaper, and A. García-Saez, Phys. Rev. Lett. 113, 257202 (2014).

[18] F. Verstraete, J. J. Garcia-Ripoll, and J. I. Cirac, Phys. Rev. Lett. 93, 207204 (2004).

[19] A. H. Werner, D. Jaschke, P. Silvi, M. Kliesch, T. Calarco, J. Eisert, and S. Montangero, Phys. Rev. Lett. 116, 237201 (2016).

[20] P. Silvi, E. Rico, T. Calarco, and S. Montangero, New J. Phys. 16, 103015 (2014).

[21] L. Tagliacozzo, A. Celi, and M. Lewenstein, Phys. Rev. X 4, 041024 (2014).

[22] T. Pichler, M. Dalmonte, E. Rico, P. Zoller, and S. Montangero, Phys. Rev. X 6, 011023 (2016).

[23] S. Singh, R. N. C. Pfeifer, and G. Vidal, Phys. Rev. A 82, 050301 (2010).

[24] S. Singh, R. N. C. Pfeifer, and G. Vidal, Phys. Rev. B 83, 115125 (2011).

[25] S. Singh and G. Vidal, Phys. Rev. B 86, 195114 (2012).

[26] A. Weichselbaum, Ann. Phys. 327, 2972 (2012).

[27] G. Vidal, Phys. Rev. Lett. 91, 147902 (2003).

[28] G. Vidal, Phys. Rev. Lett. 93, 040502 (2004).

[29] M. Levin and C. P. Nave, Phys. Rev. Lett. 99, 120601 (2007).

[30] T. Nishino and K. Okunishi, J. Phys. Soc. Jpn. 65, 891 (1996).

[31] F. Verstraete and J. I. Cirac, arXiv:cond-mat/0407066.

[32] V. Murg, F. Verstraete, and J. I. Cirac, Phys. Rev. A 75, 033605 (2007).

[33] R. Orús, Phys. Rev. B 85, 205117 (2012).

[34] H.-H. Zhao, Z.-Y. Xie, T. Xiang, and M. Imada, Phys. Rev. B 93, 125115 (2016).
[35] G. H. Golub and C. F. Van Loan, Matrix Computations (Johns Hopkins University Press, Baltimore, MA, 2012), Vol. 3.

[36] J. W. Demmel, Applied Numerical Linear Algebra (Society for Industrial and Applied Mathematics, Philadelphia, 1997).

[37] N. B. Erichson, K. Manohar, S. L. Brunton, and J. N. Kutz, arXiv:1703.09074.

[38] T. Hastie, R. Tibshirani, and J. Friedman, The Elements of Statistical Learning: Data Mining, Inference, and Prediction, 2nd ed. (Springer, New York, 2009).

[39] A. Cichocki, D. P. Mandic, A. H. Phan, C. F. Caiafa, G. Zhou, Q. Zhao, and L. D. Lathauwer, IEEE Signal Process. Mag. 32, 145 (2015).

[40] V. Vijayakumari, World J. Comp. Appl. Technol. 1, 41 (2013).

[41] A. G. Pai, S. L. Fernandes, K. Nayak, Nagesha, K. K. Accamma, K. Sushmitha, and K. Kumari, in Second International Conference on Electronics and Communication Systems (ICECS) (IEEE, New Jersey, 2015), pp. 577-582.

[42] M. E. Wall, A. Rechtsteiner, and L. M. Rocha, in A Practical Approach to Microarray Data Analysis, edited by D. P. Berrar, W. Dubitzky, and M. Granzow (Springer, Berlin, 2003), pp. 91109.

[43] A. S. Jermyn, arXiv:1709.03080.

[44] N. Halko, P.-G. Martinsson, and J. A. Tropp, SIAM Rev. 53, 217 (2011).

[45] D. Tamascelli, R. Rosenbach, and M. B. Plenio, Phys. Rev. E 91, 063306 (2015).

[46] E. Anderson, Z. Bai, C. Bischof, L. Blackford, J. Demmel, J. Dongarra, J. Du Croz, A. Greenbaum, S. Hammarling, A. McKenney, and D. Sorensen, LAPACK Users' Guide, 3rd ed. (Society for Industrial and Applied Mathematics, Philadelphia, 1999).

[47] Y.-Y. Shi, L.-M. Duan, and G. Vidal, Phys. Rev. A 74, 022320 (2006).

[48] W. Hackbusch and S. Kühn, J. Fourier Anal. Appl. 15, 706 (2009).

[49] V. Murg, F. Verstraete, O. Legeza, and R. M. Noack, Phys. Rev. B 82, 205105 (2010).

[50] N. Nakatani and G. K.-L. Chan, J. Chem. Phys. 138, 134113 (2013).

[51] M. Gerster, P. Silvi, M. Rizzi, R. Fazio, T. Calarco, and S. Montangero, Phys. Rev. B 90, 125154 (2014).

[52] M. Gerster, M. Rizzi, F. Tschirsich, P. Silvi, R. Fazio, and S. Montangero, New J. Phys. 18, 015015 (2016).

[53] C. Eckart and G. Young, Psychometrika 1, 211 (1936).

[54] L. Mirsky, Quart. J. Math. 11, 50 (1960).

[55] P.-G. Martinsson, V. Rokhlin, and M. Tygert, Appl. Comput. Harmonic Anal. 30, 47 (2011).

[56] V. Rokhlin, A. Szlam, and M. Tygert, SIAM J. Matrix Anal. Appl. 31, 1100 (2010).

[57] M. Gu, SIAM J. Sci. Comput. 37, A1139 (2015).

[58] A. Szlam, Y. Kluger, and M. Tygert, arXiv:1412.3510. 
[59] N. B. Erichson, S. Voronin, S. L. Brunton, and J. N. Kutz, arXiv:1608.02148.

[60] S. Voronin and P.-G. Martinsson, arXiv:1502.05366.

[61] P. Silvi, F. Tschirsich, M. Gerster, J. Jünemann, D. Jaschke, M. Rizzi, and S. Montangero, arXiv:1710.03733.

[62] K. A. Penson and M. Kolb, Phys. Rev. B 30, 1470 (1984).

[63] M. E. Fisher and M. N. Barber, Phys. Rev. Lett. 28, 1516 (1972).

[64] M. S. L. du Croo de Jongh and J. M. J. van Leeuwen, Phys. Rev. B 57, 8494 (1998).

[65] H. W. J. Blöte and Y. Deng, Phys. Rev. E 66, 066110 (2002).

[66] M. Rizzi, S. Montangero, P. Silvi, V. Giovannetti, and R. Fazio, New J. Phys. 12, 075018 (2010).

[67] P. Calabrese and A. Lefevre, Phys. Rev. A 78, 032329 (2008).

[68] J. Cardy, Finite-Size Scaling (Elsevier, Amsterdam, 2012), Vol. 2.

[69] P. Calabrese and J. Cardy, J. Phys. A 42, 504005 (2009).

[70] C. Musco and C. Musco, in Advances in Neural Information Processing Systems 28, edited by C. Cortes, N. D. Lawrence, D. D. Lee, M. Sugiyama, and R. Garnett (Curran Associates, Inc., New York, 2016), pp. 1396-1404.
[71] J. A. Tropp, A. Yurtsever, M. Udell, and V. Cevher, arXiv:1609.00048.

[72] J. Eisert and T. J. Osborne, Phys. Rev. Lett. 97, 150404 (2006).

[73] J. Haegeman, J. I. Cirac, T. J. Osborne, I. Pižorn, H. Verschelde, and F. Verstraete, Phys. Rev. Lett. 107, 070601 (2011).

[74] T. W. B. Kibble, J. Phys. A 9, 1387 (1976).

[75] W. H. Zurek, Nature (London) 317, 505 (1985).

[76] S. R. White, Phys. Rev. B 72, 180403 (2005).

[77] C. Hubig, I. P. McCulloch, U. Schollwöck, and F. A. Wolf, Phys. Rev. B 91, 155115 (2015).

[78] J. Jordan, R. Orús, G. Vidal, F. Verstraete, and J. I. Cirac, Phys. Rev. Lett. 101, 250602 (2008).

[79] G. Vidal, Phys. Rev. Lett. 98, 070201 (2007).

[80] R. Orús and G. Vidal, Phys. Rev. B 78, 155117 (2008).

[81] Z.-C. Gu, M. Levin, and X.-G. Wen, Phys. Rev. B 78, 205116 (2008).

[82] H. C. Jiang, Z. Y. Weng, and T. Xiang, Phys. Rev. Lett. 101, 090603 (2008).

[83] R. Orús and G. Vidal, Phys. Rev. B 80, 094403 (2009).

[84] S. Morita, R. Igarashi, H.-H. Zhao, and N. Kawashima, arXiv:1712.01458. 